13

\title{
Механизм термической ионизации уротропина на поверхности интерметаллида $\mathrm{NaAu}_{x}$
}

\author{
(C) М.В. Кнатько, М.Н. Лапушкин
}

Физико-технический институт им. А.Ф. Иофрфе РАН, 194021 Санкт-Петербург, Россия

e-mail: Mkhome2005@mail.ru

Поступило в Редакцию 12 августа 2021 г.

В окончательной редакции 24 ноября 2021 r.

Принято к публикации 1 декабря 2021 г.

Исследована термическая ионизация уротропина $\left(\mathrm{C}_{6} \mathrm{H}_{12} \mathrm{~N}_{4}\right)$ на поверхности интерметаллида $\mathrm{NaAu}_{x}$. Установлено, что термостимулированные на поверхности процессы распада, десорбции и ионизации адсорбированных соединений протекают вследствие накопления энергии на степенях свободы адсорбционного комплекса, включающего адсорбированное соединение и твердое тело, по механизму мономолекулярных реакций распада, при этом распад адсорбционного комплекса сопровождается десорбцией ионов, не находящихся в тепловом равновесии с твердым телом. Однотипность температурных зависимостей тока ионов и их распределение по двум группам позволили заключить, что с поверхности десорбируются ионы, отвечающие распадам отдельных адсорбированных молекул, а также распадам образующихся на поверхности димеров, при этом распад молекул уротропина в процессе термической ионизации происходит так же, как их распад в вакууме при электронной ионизации, что свидетельствует о сохранении объемной структуры молекул уротропина при адсорбции и значительном времени жизни возбужденного состояния соединений на $\mathrm{NaAu}_{x}$.

Ключевые слова: термическая ионизация, уротропин, интерметаллид, $\mathrm{NaAu}_{x}$.

DOI: $10.21883 /$ JTF.2022.03.52144.236-21

\section{Введение}

Интерес к изучению термической ионизации (ТИ) органических соединений определен как практической значимостью создания высокочувствительных селективных детекторов, так и возможностью изучения каталитических реакций, протекающих на поверхности твердых тел [1]. Детекторы могут применяться для нахождения в газо-воздушных смесях следов различных биологически активных веществ: лекарств, пищевых добавок и т. п. Чувствительность к парциальному давлению азотсодержащих соединений в газо-воздушной смеси при атмосферном давлении достигает рекордных значений $10^{-13}$ Torr [2], что определяет актуальность поиска новых материалов для их создания [3].

В [4] в качестве материала для эмиттера термической ионизации органических соединений предложено использовать интерметаллид $\mathrm{NaAu}_{x}$. В цикле работ $[4,5]$ показано, что эмиттер в виде ленты из золота, с созданным на ее поверхности слоем интерметаллида $\mathrm{NaAu}_{x}$, сравним по эффективности ионизации тестовых азотсодержащих молекул с высокоэффективными эмиттерами из окисленного W [3]. В [4] показано отличие каталитических свойств интерметаллидов от металлов и окисленных металлов - на поверхности $\mathrm{NaAu}_{x}$ протекают не только реакции распада, характерные для металлов и окисленных металлов, но и реакции ассоциации с образованием ионов с массой до двух раз превышающих массу адсорбируемого соединения. Изучение процессов
ТИ органических соединений на поверхности $\mathrm{NaAu}_{x}$ позволяет понять механизм взаимодействия органических соединений с поверхностью интерметаллида и природу его каталитической активности.

ТИ происходит на поверхности эмиттера, нагретого до температуры $T$, достаточной, чтобы не происходило накопления на поверхности молекул и продуктов реакций в адсорбированном слое. В этих условиях при постоянных потоке молекул $v$ и $T$ эмиттера на его поверхности устанавливается равновесная концентрация адсорбированных молекул и образующихся из них на поверхности соединений. При ТИ на металлах и окислах адсорбируемые соединения и продукты, образуемые с их участием за время жизни на поверхности, приходят в тепловое и зарядовое равновесие с эмиттером. Распределение десорбирующихся с поверхности ионов по начальным скоростям соответствует распределению Максвела с температурой эмиттера [1].

В обзоре [1] на примере аминов рассмотрен процесс адсорбции и ТИ азотсодержащих соединений. Показано, что при адсорбции аминов на металлах и окислах образуется связь неподеленной пары электронов атома $\mathrm{N}$ с поверхностью, что приводит к оттягиванию электронной плотности от атома $\mathrm{N}$ к поверхности и перераспределению электронной плотности в молекуле, вследствие чего в молекуле ослабляются $\beta$-связи по отношению к атому N. В результате ослабления этих связей происходит распад молекул (M) с образованием на поверхности $(\mathrm{M}-\mathrm{H})$ или $(\mathrm{M}-\mathrm{R})$, где 
$\mathrm{R}$ - углеводородный радикал, например, $\mathrm{CH}_{3}$ для таких соединений как диэтил- и триэтиламинамин [6]. Эти распады сопровождаются образованием двойной связи атома углерода с азотом, в результате чего $\mathrm{N}$ переходит в четырехвалентное состояние, его связь с поверхностью ослабляется, а десорбция облегчается. Кроме $(\mathrm{M}-\mathrm{H})$ и $(\mathrm{M}-\mathrm{R})$, на поверхности образуются их дегидрированные продукты. Если в результате реакций на поверхности образуется $i$ видов продуктов, которые десорбируются с величиной потока $v_{i}$, то для каждого из продуктов, десорбирующегося с поверхности, можно записать [1]:

$$
v_{i}=\gamma_{i}(T) v,
$$

где $\gamma_{i}(T)$ - индивидуальный коэффициент для $i$-ых частиц, зависящий от взаимодействующей пары адсорбируемое соединение-эмиттер, отражающий реакции в адсорбированном слое, приводящие к образованию и десорбции $i$-ой частицы. Для стационарных условий при адсорбции аминов на металлах и их окислах $\gamma_{i}(T)$, в целом, отражает диссоциацивную адсорбцию и десорбцию ее продуктов по вышеописанному механизму. Часть из этих продуктов десорбируется в ионном виде. Для отношения потоков частиц, десорбирующихся в виде заряженных частиц $\left(v_{i}^{+}\right)$и нейтральных частиц $\left(v_{i}^{0}\right)$, можно записать [1]:

$$
\frac{v_{i}^{+}}{v_{i}^{0}}=A_{i} \exp \left(\frac{1}{k T}\left(\varphi-V_{i}+e(e E)^{1 / 2}\right)\right),
$$

где $e-$ заряд электрона, $V_{i}$ - потенциал ионизации $i$-ых частиц, $\varphi-$ работа выхода поверхности, $A_{i}-$ отношение полных статистических сумм для заряженных и нейтральных частиц, $k-$ постоянная Больцмана, $E$ - напряженность электрического поля у поверхности эмиттера.

В случае гидразинов, гидразонов, азидов и тетразенов за счет частичной гибридизации электронов в группе атомов азота перераспределение электронной плотности при адсорбции в этом ряду уменьшается, вследствие чего наблюдается образование молекулярного иона $[\mathrm{M}]^{+}$ и уменьшение эффективности образования $(\mathrm{M}-\mathrm{H})$ и $(\mathrm{M}-\mathrm{R})[1]$. В случае тетраметилтетразена с поверхности окисла $\mathrm{W}$ десорбируется только $[\mathrm{M}]^{+}[7]$. В соответствии с основными правилами ТИ азотсодержащих молекул на металлах и окислах, сформулированными в [1], с поверхности в виде ионов преимущественно десорбируются соединения, в которых атом $\mathrm{N}$ находится в четырехвалентном состоянии, а остальные связи химически насыщены, величина $\left(\varphi-V_{i}\right)$ этих соединений при температуре эмиттера должна обеспечивать заметную эффективность ионизации по (2).

Температурные зависимости токов ионов при ТИ органических соединений на окислах $\mathrm{W}$ имеют в основном колоколообразный вид $[1,6]$. В [8] колоколообразный вид температурных зависимостей тока ионов связали с зависимостью от температуры удельной скорости мономолекулярного распада адсобированных молекул на поверхности $\gamma_{i}(T)$. Для определения этой зависимости использован формализм теории мономолекулярного распада в газе [9] с активацией адсорбированных молекул за счет их взаимодействия с нагретым твердым телом эмиттером ионов. Активация и дезактивация молекул происходит при статистически редких больших амплитудах колебаний атомов поверхности, колебания атомов поверхности со средними амплитудами не участвуют в процессе [10]. Считая, что по всем каналам распада $\gamma_{i}<1$ и распады не конкурируют между собой, в $[8,10]$ предложена зависимость $\gamma_{i}(T)$ в виде

$$
\begin{aligned}
\gamma_{i}(T)= & \operatorname{const}\left(E_{a i} / k T\right)^{b} \exp \left(-E_{a i} / k T\right) \\
& =\operatorname{const}(1 / k T)^{b} \exp \left(-E_{a i} / k T\right),
\end{aligned}
$$

где $E_{a i}$ - энергия активации распада молекул с образованием $i$-го продукта, $b-$ число активных по отношению к диссоциации колебательных степеней свободы в молекуле. Здесь и далее величина const включает все параметры, не зависящие от переменных в экспериментах, таких как $v, T$ и $E$. Плотность ионного тока $j_{i}(T)$ при ТИ для $i$-го продукта из $(1)-(3)$ в соответствии с [8] может быть записана, как

$$
\begin{aligned}
& j_{i}(T)=\operatorname{const} v(1 / k T)^{b} \\
& \times \exp \left(\frac{1}{k T}\left(\varphi-V_{i}+e(e E)^{1 / 2}\right)-E_{a i} / k T\right) .
\end{aligned}
$$

В (4) экспоненциальный член отвечает за рост плотности ионного тока с ростом $T$, а предэкспоненциальный член $(1 / k T)^{b}$ отвечает за падающую часть температурной зависимости ионного тока, что делает эту зависимость отвечающей виду наблюдаемых колоколобразных температурных зависимостей $j_{i}(T)$. Однако проверка пригодности выражения (4) для аппроксимации температурных зависимостей ионных токов на примере зависимости тока ионов в [8] при подборе параметров $b$ и $E_{a i}$ показывает сходимость экспериментальных данных с выражением (4) только вблизи максимума тока ионов (не более $50 \%$ от максимального значения тока).

Экспериментальные исследования зависимости $\gamma(T)$ диссоциативной адсорбции простых молекул также не подтвердили справедливость выражения (3). В [11] для диссоциативной адсорбции водорода на поверхности $\mathrm{Cu}(111)$ измерена $\gamma(T)$ в диапазоне температур металла от 300 до $2000 \mathrm{~K}$. В этом диапазоне температур $\gamma$ изменяется на 14 порядков величины, при этом зависимость $\gamma(T)$ следует уравнению Аррениуса для скорости простой химической реакции $\gamma(T)=$ const $\cdot \exp \left(-E_{a} / k T\right)$.

При статистическом рассмотрении мономолекулярного распада сложных молекул в газе как макроскопических систем, при высокой частоте соударений молекул (активации и деактивации) получено, что удельная скорость реакции от $T$ определяется уравнением Аррениуса, а величина $E_{a}$ определяется эффективной энергией активации молекул [9]. В случае адсорбированных молекул это означает, что их взаимодействие 
с поверхностью (активация-деактивация) в отличие от предположения в [10] происходит с большой частотой, соответствующей частоте колебаний адсорбированных молекул и колебаний атомов решетки твердого тела.

Для многоатомных молекул $\gamma(T)$ экспоненциально растет с ростом $T$, однако отмечается отклонение от закона Аррениуса. Например, для диссоциативной адсорбции метана на $\operatorname{Pt}(111)$ [12] показано, что зависимость $\gamma(T)$, измеренная в диапазоне пяти порядков величины, в координатах $\ln (\gamma)$ и $1 / k T$ отклоняется от линейной зависимости. Это может свидетельствовать о более сложном характере взаимодействия адсорбированных молекул с поверхностью, чем в моделях взаимодействия молекул в газах.

Энергия активации молекул при диссоциативной адсорбции складывается, в основном, из энергии молекул до адсорбции и энергии, передаваемой нагретым твердым телом адсорбированным молекулам, а активированное состояние может реализоваться как диссоциацией адсорбированных молекул, так и их десорбцией $[11,12]$. Это дает основание рассматривать распад не отдельной молекулы, а адсорбционного комплекса, включающего адсорбированную молекулу и твердое тело. Активированное состояние адсорбционного комплекса реализуется двумя типами распадов: десорбцией молекул и распадом молекул с образованием адсорбированных продуктов. При адсорбции органических молекул активированное состояние может реализовываться большим количеством распадов. В [13] исследован процесс термического разложения метиламина $\left(\mathrm{CH}_{3} \mathrm{NH}_{2}\right)$ на $\mathrm{Pd}(111)$ и установлено, что адсорбированные молекулы диссоциируют с образованием $\mathrm{CH}_{3} \mathrm{NH}$ и Н. В результате дегидрирования метиламина связь молекул с поверхностью неподеленной парой электронов атомов $\mathrm{N}$ переходит в химическую связь. За счет сильной адсорбционной связи молекулы не десобируются с поверхности, вследствие чего продолжается их термический распад до образования CN. Тогда в соответствии с правилами ТИ [1] при адсорбции метиламина не должно наблюдаться десорбции ионов. Расчет плотности методом функционала [14] показал, что при термической диссоциации адсорбированного метиламина на металле на первом шаге дегидрирования может образовываться $\mathrm{CH}_{2} \mathrm{NH}_{2}$. Свободная связь углерода группы $\mathrm{CH}_{2}$ может привести к дополнительной связи адсорбированной молекулы с поверхностью или создать связь с атомом азота, переведя его в четырехвалентное состояние, как это характерно для ТИ аминов. В случае образования связи углерода с поверхностью десорбция и ионизация дегидрированного метиламина маловероятна. При образовании двойной связи углерода с атомом $\mathrm{N}$ с поверхности могут десорбироваться механизмом ТИ ионы $[\mathrm{M}-\mathrm{H}]^{+}$. При ТИ метиламина на окисленном рении [15] с поверхности десорбируются ионы $[\mathrm{M}-\mathrm{H}]^{+}$. Это демонстрирует, что на поверхности может параллельно происходить ряд распадов и только некоторые из них приводят к ТИ.
Активированное состояние молекул метиламина может реализоваться деактивацией, десорбцией и тремя путями диссоциации с образованием на поверхности $\mathrm{CH}_{3}-\mathrm{NH}$ [14], $\mathrm{CH}_{2}-\mathrm{NH}_{2}$ и $\mathrm{CH}_{2}=\mathrm{NH}_{2}$ [14]. Только $\mathrm{CH}_{2}=\mathrm{NH}_{2}$ из трех возможных продуктов распада по правилам ТИ может десорбироваться в виде ионов $[\mathrm{M}-\mathrm{H}]^{+}$. В этом случае $\gamma(T)$ ТИ $(\mathrm{M}-\mathrm{H})$ отражает конкуренцию между десорбцией и диссоциацией с образованием трех возможных продуктов.

Эффективность ТИ азотсодержащих соединений на поверхности интерметаллида $\mathrm{NaAu}_{x}$ сравнима с окисленным W [4], но масс-спектр их ТИ на $\mathrm{NaAu}_{x}$ имеет большее количество линий по сравнению с масс-спектром ТИ на поверхности металлов и окислов металлов $[1,4]$. Часть линий в масс-спектрах ТИ на $\mathrm{NaAu}_{x}$ азотсодержащих соединений соответствует ассоциативным соединениям с массой до двух раз большей, чем у адсорбируемого соединения. Для определения различий в формировании масс-спектров ТИ на $\mathrm{NaAu}_{x}$ и на гокисленном W было выполнено детальное исследование ТИ тетраметилтетразена $\mathrm{C}_{4} \mathrm{H}_{12} \mathrm{~N}_{4}$ (TMT) на $\mathrm{NaAu}_{x}[16]$. Интерес к ТМТ вызван тем, что при его ТИ на окисленном $\mathrm{W}$ с поверхности десорбируются только молекулярные ионы [7], а на $\mathrm{NaAu}_{x}$ масс-спектр содержит несколько десятков линий, включая ионы ассоциативных соединений [16].

В [16] установлено, что в отличие от окисленного W на поверхности $\mathrm{NaAu}_{x}$ происходит диссоциативная адсорбция ТМТ с распадом ТМТ по двойной связи группы атомов азота $\mathrm{N}-\mathrm{N}=\mathrm{N}-\mathrm{N}$. В результате диссоциативной адсорбции ТМТ на поверхности образуются радикалы $\left(\mathrm{CH}_{3}\right)_{2}=\mathrm{N}-\mathrm{N}$. Взаимодействие этих радикалов между собой приводит к образованию гетероциклических соединений, которые в процессе десорбции с поверхности ионизуются, образуя масс-спектр ТИ. В результате этого в масс-спектре термической ионизации ТМТ отсутствуют молекулярные ионы, а также ионы $[\mathrm{M}-\mathrm{H}]^{+}$ и $[\mathrm{M}-\mathrm{R}]^{+}$, характерные для ТИ аминов, гидразинов и гидразонов. Температурные зависимости тока ионов при термической ионизации ТМТ на $\mathrm{NaAu}_{x}$ для разных ионов имеют разный вид: от пороговых зависимостей и монотонно растущих с температурой до колоколообразных. Такое разнообразие температурных зависимостей тока ионов отражает многообразие реакций в адсорбированном слое.

Уротропин $\mathrm{C}_{6} \mathrm{H}_{12} \mathrm{~N}_{4}$, как и тетраметилтетразен, содержит 4 атома $\mathrm{N}$, но в отличие от „плоской“ структуры ТМТ уротропин - неправильный декаэдр, в четырех вершинах которого расположены атомы азота. Учитывая пространственную структуру молекул, можно было ожидать, что адсорбция уротропина происходит путем установления связи с поверхностью неподеленной парой электронов одного из атомов азота, как для азотсодержащих соединений [17].

На основании сравнения масс-спектров и анализа схем распадов уротропина при ТИ на эмиттере из $\mathrm{NaAu}_{x}$, нагретого до $T>800 \mathrm{~K}$, и ионизации электронами в [18] 
сделан вывод, что адсорбция уротропина на $\mathrm{NaAu}_{x}$, в отличие от адсорбции ТМТ, происходит без существенного изменения его структуры. Это позволяет ожидать меньшего, чем в случае ТМТ, разнообразия реакций в адсорбированном слое и дает возможность более детального изучения взаимодействия адсорбируемых молекул с поверхностью интерметаллида $\mathrm{NaAu}_{x}$. С этой целью выполнены новые детальные исследования температурных зависимостей тока, образующихся при ТИ уротропина ионов, а также получено распределение по кинетическим энергиям десорбирующихся ионов.

\section{1. Эксперимент}

Работа выполнена в 90-градусном секторном магнитном масс-спектрометре с радиусом $300 \mathrm{~mm}$ со сдвоенным источником ионов, получаемых термической ионизацией (ТИ) и электронной ионизацией (ЭИ). Источники ионов расположены по оси движения ионов от эмиттера ТИ через источник ЭИ к электромагниту. Сдвоенный источник ионов позволяет проводить анализ десорбирующихся с эмиттера ионов по кинетическим энергиям методом кривых задержки ионов, как это представлено в [19]. Для реализации метода кривых задержки на электроды источника ЭИ подавался задерживающий потенциал.

Для напыления на эмиттер потока атомов натрия использовали ячейку Кнудсена. Натрий получали разложением смеси бихромата натрия с цирконием. Для ввода паров и газов в масс-спектрометр использовалась высоковакуумная многоканальная система напуска. Кислород в системе напуска получали термическим разложением $\mathrm{KMnO}_{4}$. Чистота газа была определена масс-спектрометрически и составляла 99.99. В работе использован уротропин производства ПАО „Метафракс“ марки СВ чистотой не хуже 99.5\%. Перед вводом уротропина в систему напуска для обезвоживания и дегазации образца уротропина проводилась его вакуумная тренировка путем замораживания и нагрева под вакуумной откачкой.

Эмиттером ТИ в масс-спектрометре служила золотая лента со сформированой на ней пленкой сплава $\mathrm{NaAu}_{x}$. Золотая лента вырезана из золотой фольги чистотой 99.99 с размерами $0.05 \times 2.5 \times 50 \mathrm{~mm}$. Перед исследованиями золотая лента была отожжена в течение $10 \mathrm{~h}$ в вакууме $1 \cdot 10^{-8}$ Torr при $T=1100 \mathrm{~K}$, а затем в течение $30 \mathrm{~h}$ - в кислороде при $2 \cdot 10^{-6}$ Torr. Формирование слоя $\mathrm{NaAu}_{x}$ производили in situ. Для образования пленочного покрытия эмиттера слоем $\mathrm{NaAu}_{x}$ на золотую ленту при $T=1100 \mathrm{~K}$ напыляли натрий из ячейки Кнудсена. Эмиттер нагревался прямым накалом, его температура в диапазоне 970-1100 K измерялась с помощью оптического микропирометра „Руго“ производства Pyrometer Instrumental Company с погрешностью менее $\pm 5^{\circ}$. В диапазоне $800-970 \mathrm{~K}$ температура определялась по интерполяции градуировки температуры по подводи- мой мощности к ленте, выполненной в диапазоне температур эмиттера 970-1100 K. Погрешность определения температуры в диапазоне $800-970 \mathrm{~K}$ составляла $\pm 10 \mathrm{~K}$.

Для очистки поверхности эмиттера от возможного загрязнения продуктами распада уротропина совместно с напуском уротропина в масс-спектрометр напускался кислород до давления $1 \cdot 10^{-6}$ Torr. Как показали эксперименты, кислород не оказывал влияния на ТИ уротропина, но обеспечивал очистку эмиттера от углеродных загрязнений. Напряженность электрического поля в промежутке лента-вытягивающий электрод не превышала $200 \mathrm{~V} / \mathrm{cm}$, что позволяло исключить влияние слагаемого $e(e E)^{1 / 2}$ в (2), которое не превышало $0.005 \mathrm{eV}$, а также считать, что поле пятен на поверхности с разными величинами $\varphi$ не скомпенсировано, поверхность проявляет в эмиссионных процессах единое эффективное значение $\varphi[1]$.

\section{2. Результаты и обсуждение}

На рис. 1 приведен масс-спектр ТИ уротропина на $\mathrm{NaAu}_{x}$ при $T=860 \mathrm{~K}$, из которого видно, образование молекулярных ионов $[\mathrm{M}]^{+}$с $m / z 140$ и отсутствие масс-линий, соответствующих $[\mathrm{M}-\mathrm{H}]^{+}$с $m / z 139$ и $\left[\mathrm{M}-\mathrm{CH}_{3}\right]^{+} \mathrm{c} m / z 125$, которые должны быть основными в масс-спектре азотсодержащих соединений, как это следует из результатов исследований ТИ органических соединений, приведенных в обзоре [1]. Форма линий в масс-спектре хорошо описывается функцией Гаусса с полной шириной линии на полувысоте, которая составляет величину, например, для $m / z$, равную 0.3 .

В соответствии с выводами [1] об уменьшении роли неподеленной пары электронов $\mathrm{N}$ при адсорбции соединений с кратным количеством атомов $\mathrm{N}$, можно заключить, что значительного перераспределения электронной плотности в молекулах уротропина при адсорбции на $\mathrm{NaAu}_{x}$ не происходит. При этом наличие

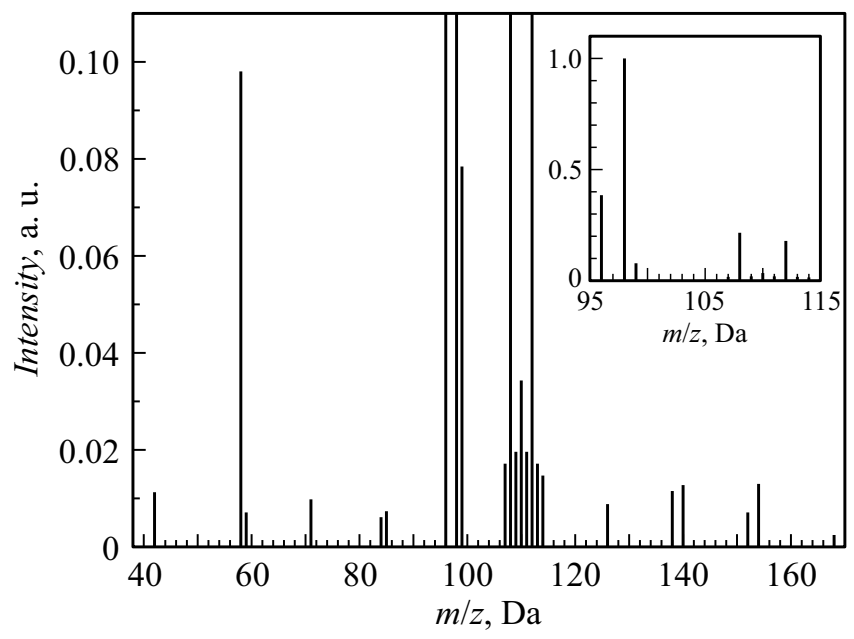

Рис. 1. Масс-спектр термической ионизации уротропина. Температура эмиттера $860 \mathrm{~K}$. 


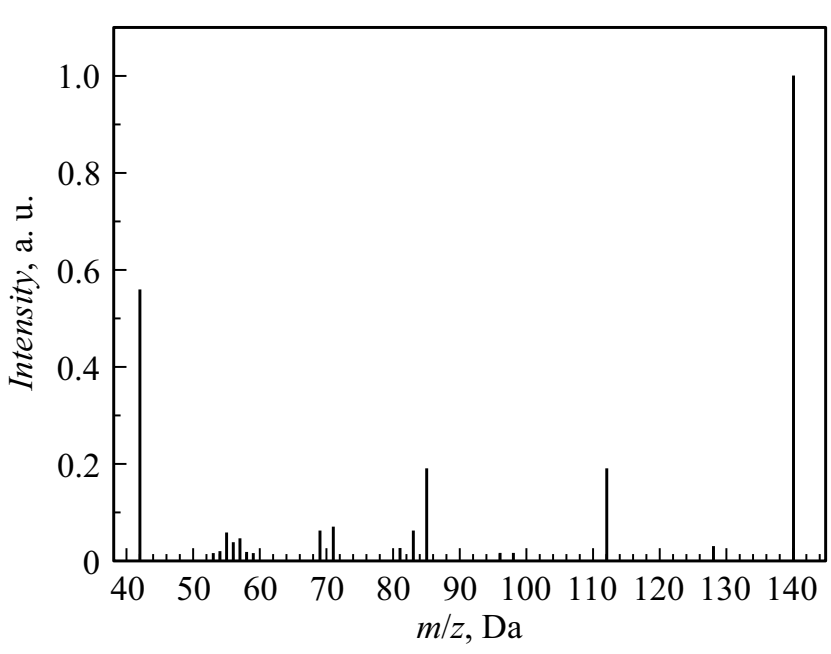

Рис. 2. Масс-спектр электронной ионизации уротропина. Энергия ионизирующих электронов $70 \mathrm{eV}$.

многочисленных линий в спектре ТИ свидетельствует о реакциях уротропина в адсорбированном слое, приводящих к образованию ТИ продуктов, не характерных для ТИ азотсодержащих молекул на металлах и их окислах.

В [18] установлена корреляция между масс-спектром ТИ уротропина на $\mathrm{NaAu}_{x}$ при температуре эмиттера $T>800 \mathrm{~K}$ и масс-спектром ЭИ при энергии электронов $70 \mathrm{eV}$. Сравнивая рис. 1 и 2, на котором представлен масс-спектр ЭИ уротропина при энергии электронов $70 \mathrm{eV}$, можно видеть, что масс-спектры содержат $[\mathrm{M}]^{+}$ и группы линий вблизи $m / z, 126,112,96-98,85,58$ и 42. Те же группы линий получены в масс-спектре ЭИ уротропина в [20] при энергии электронов $70 \mathrm{eV}$.

B [21] на примере триэтиламина проведено сопоставление схем образования положительных ионов при ТИ с температурой эмиттера $T>700 \mathrm{~K}$ [6] и при ионизации электронами с энергией электронов $30 \mathrm{eV}$. В результате сопоставления показано, что при разных видах ионизации соединения проходят через одни и те же возбужденные состояния, приводящие к подобным распадам. Учитывая выводы работы [21], можно полагать что уротропин, адсорбированный на поверхности $\mathrm{NaAu}_{y}$, нагретой до температуры $T>800 \mathrm{~K}$, претерпевает те же распады, что и при его возбуждении в процессе ЭИ с энергией электронов $70 \mathrm{eV}$.

В [20] на основе анализа масс-спектров изотопов уротропина предложена схема формирования масс-спектра ЭИ при энергии электронов $70 \mathrm{eV}$, согласно которой ионы уротропина $[\mathrm{M}]^{+}$распадаются в следующей последовательности:

$$
\begin{aligned}
& \text { 1-я фаза распада : } \quad\left[\mathrm{C}_{6} \mathrm{H}_{12} \mathrm{~N}_{4}\right]^{+}(m / z \text { 140 }) \\
& \rightarrow\left[\mathrm{C}_{5} \mathrm{H}_{10} \mathrm{~N}_{3}\right]^{+}(m / z 112)+\mathrm{CH}_{2} \mathrm{~N}, \\
& \text { 2-я фаза распада : } \quad\left[\mathrm{C}_{5} \mathrm{H}_{10} \mathrm{~N}_{3}\right]^{+}(m / z \text { 112 }) \\
& \rightarrow\left[\mathrm{C}_{4} \mathrm{H}_{9} \mathrm{~N}_{2}\right]^{+}(m / z 85)+\mathrm{CHN},
\end{aligned}
$$

$$
\begin{aligned}
& \text { 3-я фаза распада : } \quad\left[\mathrm{C}_{4} \mathrm{H}_{9} \mathrm{~N}_{2}\right]^{+}(m / z 85) \\
& \rightarrow\left[\mathrm{C}_{2} \mathrm{H}_{4} \mathrm{~N}\right]^{+}(m / z 42)+\mathrm{C}_{2} \mathrm{H}_{4} \mathrm{NH} .
\end{aligned}
$$

В результате этих распадов образуются ионы, содержащие атомы азота в четырехвалентном состоянии. Валентности продуктов распада насыщенные. При этом образующиеся в фазах 1 и 2 распадов ионы сохраняют трехмерную структуру. В масс-спектре ТИ уротропина также наблюдаются ионы с $m / z$ 140, 112, 85 и 42.

Энергии электронов при ЭИ достаточно для ионизации молекул и их возбуждения. В вакууме отсутствуют столкновения возбужденных молекул с другими частицами, в результате чего энергия возбуждения сохраняется в продуктах распада, обеспечивая предложенную в [20] последовательность распадов. Как было показано выше, мономолекулярный распад на поверхности происходит при высокой частоте взаимодействий между адсорбированным соединением и поверхностью, т. е. обмен энергий между адсорбированным соединением и твердым телом (активация и деактивация молекул) происходит с большой частотой. Активированное состояние адсорбционного комплекса приводит либо к десорбции молекулы, либо к распаду молекулы с образованием адсорбционных комплексов продуктами распада. Образуемые продуктами распада адсорбционные комплексы при большой частоте взаимодействия с твердым телом не могут сохранить энергию активации породившего их распада. Поэтому в отличие от распадов в вакууме каждый этап распада на поверхности необходимо рассматривать как распад адсорбционного комплекса каждого продукта первичного распада. Можно предположить, что с поверхности десорбируются ионы $[\mathrm{M}]^{+}$, и их дальнейшие распады происходят вне поверхности в соответствии с предложенной в [20] последовательностью распадов за счет энергии, запасенной на поверхности. Ионы, образующиеся вне поверхности в результате распадов первичных ионов - осколочные ионы. При распадах первичных ионов в зоне ускорения электрическим полем источника ионов осколочные ионы регистрируются в виде линий, растянутых в сторону меньших масс, а при распадах в бесполевом пространстве масс-спектрометpa - в виде диффузных линий с дробным показателем массы и шириной на полувысоте в несколько масс $[1,6]$. Так как все интенсивные линии в масс-спектре являются узкими и хорошо описываются функцией Гаусса, из этого следует, что масс-спектр ТИ уротропина на $\mathrm{NaAu}_{x}$ составляют первичные ионы, отвечающие соединениям, происходящим из реакций на поверхности и ионизирующимся в процессе десорбции. На этом основании можно ожидать, что температурные зависимости тока ионов, образующихся вследствие распада адсорбционного комплекса уротропина и адсорбционных комплексов продуктов его распада, будут различаться. В ряде работ по исследованию ТИ органических соединений на окислах $\mathrm{W}$, например, [22], принято, что величина $\gamma_{i}$ слабо зависит от $T$, что позволяло из (2) по температурным зависимостям ионных токов определять значение $\left(\varphi-V_{i}\right)$. 


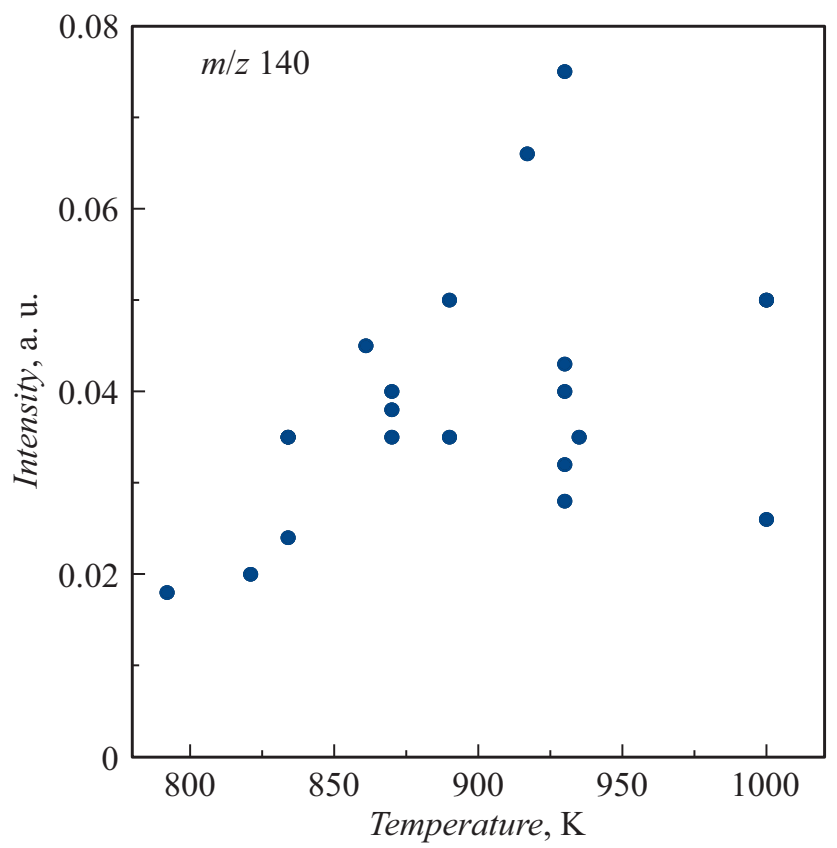

Рис. 3. Зависимость тока молекулярных ионов уротропина $[\mathrm{M}]^{+}$от температуры.

На рис. 3 показана зависимость тока молекулярных ионов уротропина $[\mathrm{M}]^{+}$от температуры эмиттера, из которого видно, что до $T=870 \mathrm{~K}$ с ростом $T$ ионный ток растет, а затем наблюдается его слабое падение. Вид этой зависимости подобен температурным зависимостям для так называемой ,легкой ионизации“ $(V<\varphi)[1,23]$. Первоначальный рост ионного тока для „легкой ионизации“ связан с ростом при уменьшении покрытия поверхности адсорбированными соединениями с ростом $T$ [23]. В процессе увеличения с ростом $T$ достигается температура эмиттера $T_{\max }$, при которой $\varphi(T)=V$, и дальнейший рост $\varphi$ в соответствии с (2) приводит к уменьшению тока ионов. Кроме того, при $\varphi>V$ по (2) эффективность ионизации уменьшается с ростом $T$. Если температурная зависимость молекулярного ионного тока уротропина $[\mathrm{M}]^{+}$отвечает случаю „легкой ионизации“, то величина $\varphi$ поверхности $\mathrm{NaAu}_{x}$ должна быть больше потенциала ионизации уротропина $V=8.5 \mathrm{eV}[24]$, т.е. $\varphi>8.5 \mathrm{eV}$. В [18] методом полного тока величина работы выхода $\mathrm{NaAu}_{x}$ была оценена как 7.6-7.9 eV. При этом для всех известных эмиттеров величины работы выхода не превышают $7 \mathrm{eV}$ [25]. Причина, приводящая к завышению оценки $\varphi$ из (2), будет обсуждена ниже.

Полагая, что температурная зависимость тока ионов определяется не только эффективностью ионизации по (2), рассмотрим процессы, которые могут на нее влиять. Для адсорбированных молекул мы рассматриваем десорбцию и диссоциацию молекул как реакции мономолекулярного распада адсорбционного комплекса. При стационарных условиях на поверхности эмиттера с температурой $T$ часть адсорбционных комплексов со скоростью $K_{0}$ переходит в активированное состояние, которое может деактивироваться со скоростью $K_{1}$. Со скоростью $K_{2}$ молекулы могут десорбироваться, со скоростью $K_{3}$ - диссоциировать с образованием $\mathrm{C}_{5} \mathrm{H}_{10} \mathrm{~N}_{3}$ в соответствии с первой фазой (5) и десорбцией продукта диссоциации, со скоростью $K_{4}-$ диссоциировать с адсорбцией $\mathrm{C}_{5} \mathrm{H}_{10} \mathrm{~N}_{3}$ на поверхности, со скоростью $K_{5}-$ диссоциировать с образованием соединений, не учтенных в схеме распадов (5) и их десорбции с поверхности, со скоростью $K_{6}$ - диссоциировать с образованием соединений, не учтенных в схеме распадов (5), с их адсорбцией на поверхности.

По теории мономолекулярного распада [9] в реакциях распада участвуют только соединения, находящиеся в активированном состоянии, т.е. соединения, имеющие внутреннюю энергию, превышающую эффективную энергию реакции. В стационарных условиях при $T=$ const относительное число молекул уротропина в составе активированных комплексов $\mathrm{N}^{*} / \mathrm{N}$ определяется отношением скоростей активации и деактивации $K_{0}$ и $K_{1}$. Количество поступающих в единицу времени молекул уротропина равно сумме деактивирующихся, десорбирующихся и распадающихся по различным каналам молекул, т.е. $v=\mathrm{N} \sum K_{i}$, где $i-$ целое число, в рассмотренном случае меняющееся от 1 до 6. Уравнение (1) можно переписать в виде $v_{i}=v\left(K_{i} / \sum K_{i}\right)$. Учитывая (2), при $V_{i}>\varphi$ можно записать для плотности ионного тока

$$
j_{i}(T)=\operatorname{const} v\left(K_{i} / \sum K_{i}\right) \exp \left(\left(\varphi-V_{i}\right) / k T\right) .
$$

$K_{i}(T)$, как это рассматривалось выше, - функции, экспоненциально растущие с температурой, которые можно аппроксимировать уравнением Аррениуса, и, следовательно, функция $K_{i} / \sum K_{i}$ при наличии нескольких каналов распада убывает с ростом $T$. При ТИ диэтил- и триэтиламина на окисленном W [6] после достижения эмиттером температуры, достаточной для активации образования и десорбции $(\mathrm{M}-\mathrm{H})$, ток ионов $[\mathrm{M}-\mathrm{H}]^{+}$ растет с температурой по (8). Активация второго канала распада с образованием $\left(\mathrm{M}-\mathrm{CH}_{3}\right)$ увеличивает $\sum K_{i}(T)$, что приводит к падению тока $[\mathrm{M}-\mathrm{H}]^{+}$. В результате температурная зависимость тока ионов $[\mathrm{M}-\mathrm{H}]^{+}$имеет колоколообразный вид $[6,8]$.

Первоначальный рост тока молекулярных ионов уротропина $[\mathrm{M}]^{+}$с $T$ по (8) связан с увеличением эффективности образования, десорбции и ионизации молекул. При достижении эмиттером температуры $T_{\max }=870 \mathrm{~K}$ активируются определенные каналы распада молекул уротропина, что приводит к прекращению роста тока и его дальнейшему падению.

Распад ионов уротропина при его ЭИ на первой фазе (5) приводит к образованию $\left[\mathrm{C}_{5} \mathrm{H}_{10} \mathrm{~N}_{3}\right]^{+}(\mathrm{m} / \mathrm{z}$ 112). При ТИ уротропина, как видно из рис. 1 , вместе с ионами $m / z 112$ регистрируются ионы с $m / z$ от 107 до 114. Образование этой группы ионов происходит при распаде адсорбированного уротропина, так же как и 


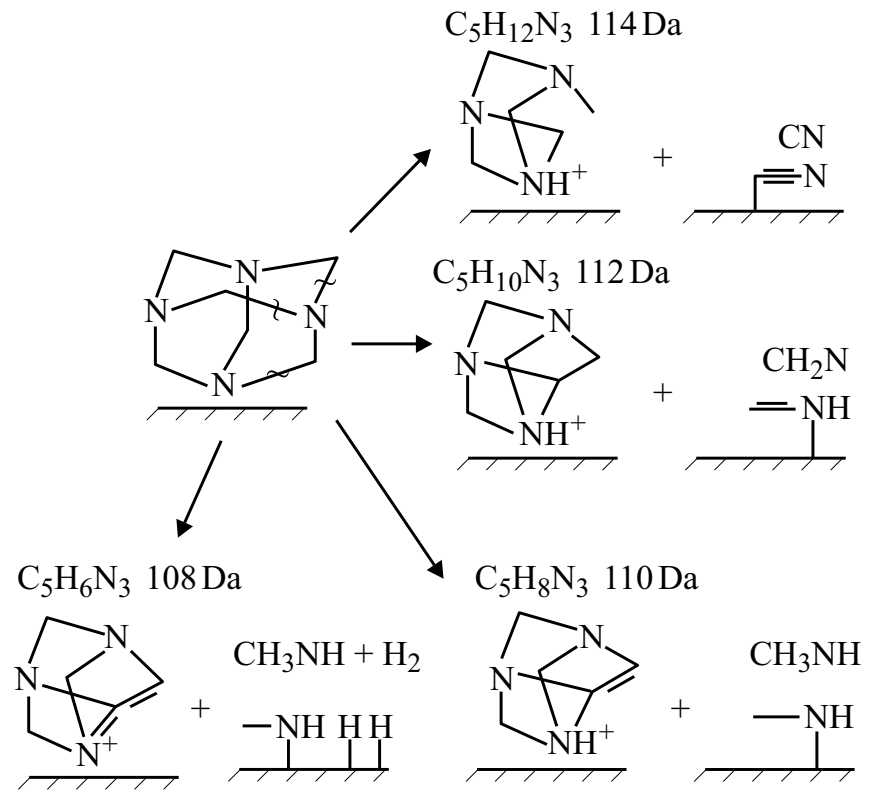

Рис. 4. Схема образования соединений на поверхности $\mathrm{NaAu}_{x}$, приводящих к образованию ионов $m / z$ 108, 110, 112 и 114.

при распаде иона уротропина при ЭИ. На основе результатов исследований [20] составлена схема распадов уротропина, показанная на рис. 4, с образованием соединений, которые при ТИ образуют основные масс-линии этой группы ионов. Как видно из рис. 4, образование этих соединений происходит при разрыве трех связей в молекуле уротропина. Продукты распада отличаются распределением между ними атомов Н. Атом азота в ионизирующихся продуктах распада находится в четырехвалентном состоянии, химические связи насыщены.

Температурные зависимости тока ионов наиболее интенсивных масс-линий этой группы ионов с $m / z 108$, 110 и 112 показаны на рис. 5. Видно, что эти зависимости похожи, что подтверждает выводы работы [26] о близости между собой температурных зависимостей тока ионов при ТИ продуктов распада, отличающихся количеством атомов водорода. Вид температурных зависимостей тока ионов с $m / z$ 108, 110 и 112 по форме соответствует температурной зависимости тока $[\mathrm{M}]^{+}$. Из сравнения рис. 3 и 5 видно, что температура начала регистрации тока ионов с $m / z 108,110$ и 112 выше, чем у молекулярных ионов, и составляет $T=820 \mathrm{~K}$. Первоначальный рост тока группы ионов с $m / z 108$, 110 и 112 при увеличении $T$ эмиттера связан по (8) с ростом эффективности образования и десорбции $K_{3}(T)$, а также с увеличением эффективности ионизации десорбирующихся соединений. При достижении температуры эмиттера $T_{\max }=920 \mathrm{~K}$ рост тока прекращается, а при $T>T_{\max }$ начинается падение тока ионов с дальнейшим ростом $T$. Из сравнения рис. 3 и 5 видно, что значения $T_{\max }$ для тока этой группы ионов более высокие, чем для молекулярных ионов. Причины расхождения этих температур будут рассмотрены ниже. Температурные зависимости тока ионов этой группы с $m / z 107$ и 114, не показанные на рис. 5 , соответствуют таковым ионов с $m / z 108,110$ и 112.

Другая группа ионов в масс-спектре ТИ уротропина с $m / z 96$ и 98. Хотя эта группа ионов регистрируется в масс-спектре ЭИ, ее образование не рассмотрено в [20]. Однако мы полагаем, что распад происходит по той же схеме, что и рассмотренный распад с образованием ионов с $m / z 108,110$ и 112 за счет разрыва трех связей в молекуле уротропина, как показано на рис. 6 .

На рис. 5 показаны температурные зависимости тока ионов с $m / z 96$ и 98. Они близки между собой, что подтверждает единый механизм их образования. Вид температурных зависимостей этой группы подобен виду температурных зависимостей молекулярных ионов и ионов группы с $m / z 107-114$. При этом температур начала регистрации тока и $T_{\max }$ для ионов с $m / z 96$ и 98 близки к таковым для молекулярных ионов, и ниже чем у группы ионов с $m / z$ 107-114.

В соответствии с предложенной в [20] последовательностью распадов ионов уротропина, при ЭИ на второй фазе происходит распад иона с $m / z 112$ с образованием иона $\left[\mathrm{C}_{4} \mathrm{H}_{9} \mathrm{~N}_{2}\right]^{+} \mathrm{c} m / z 85$, как это показано в уравнении (6). Как видно из структуры соединения $\mathrm{C}_{5} \mathrm{H}_{10} \mathrm{~N}_{3}$ (112 Da) на рис. 4, образование соединения $\mathrm{C}_{4} \mathrm{H}_{9} \mathrm{~N}_{2}(85 \mathrm{Da})$ происходит при разрыве трех связей и отделении CHN.

Образование относительно интенсивной масс-линии с $m / z 58$ в работе [20] не рассмотрено. Предполагая сохранение последовательности распадов, можно полагать, что соединение, ионизирующееся с $m / z$, образуется вследствие распада $\mathrm{C}_{4} \mathrm{H}_{9} \mathrm{~N}_{2}(85 \mathrm{Da})$ в результате разрыва трех связей и отделения CHN. На третьей фазе распада ионов уротропина при его ЭИ в соответствии с уравнением (7) образуется соединение $\mathrm{C}_{2} \mathrm{H}_{4} \mathrm{~N}$, которое при ТИ может образовывать ионы с $m / z$ 42. Эта линия наблюдается в масс-спектре ТИ уротропина, как это видно на рис. 1, что подтверждает возможность этого распада на поверхности.

Температурные зависимости тока ионов с $m / z$ 42, 58 и 85 подобны температурным зависимостям тока группы ионов с $m / z 107-114$, представленным для основных ионов на рис. 5. У них близки температуры начала регистрации и $T_{\max }$, что подтверждает общность их происхождения и корректность предложенной в [20] схемы распада ионов уротропина. При этом форма масс-линий этих ионов свидетельствует о том, что они десорбируются с поверхности, а не образуются из ионов с $m / z 112$ вне поверхности.

В масс-спектре ТИ уротропина (рис. 1) регистрируются линии, отсутствующие в масс-спектре ЭИ, а именно линии с $m / z 138,152,154$ и 168. Регистрация ионов, отвечающих соединениям с массой, больше, чем у молекул уротропина (140 Da), свидетельствует об образовании на поверхности ассоциативных соединений. О возможности реакций ассоциации между коадсорбированными органическими соединениями сообщалось в [27]. Механизм их 

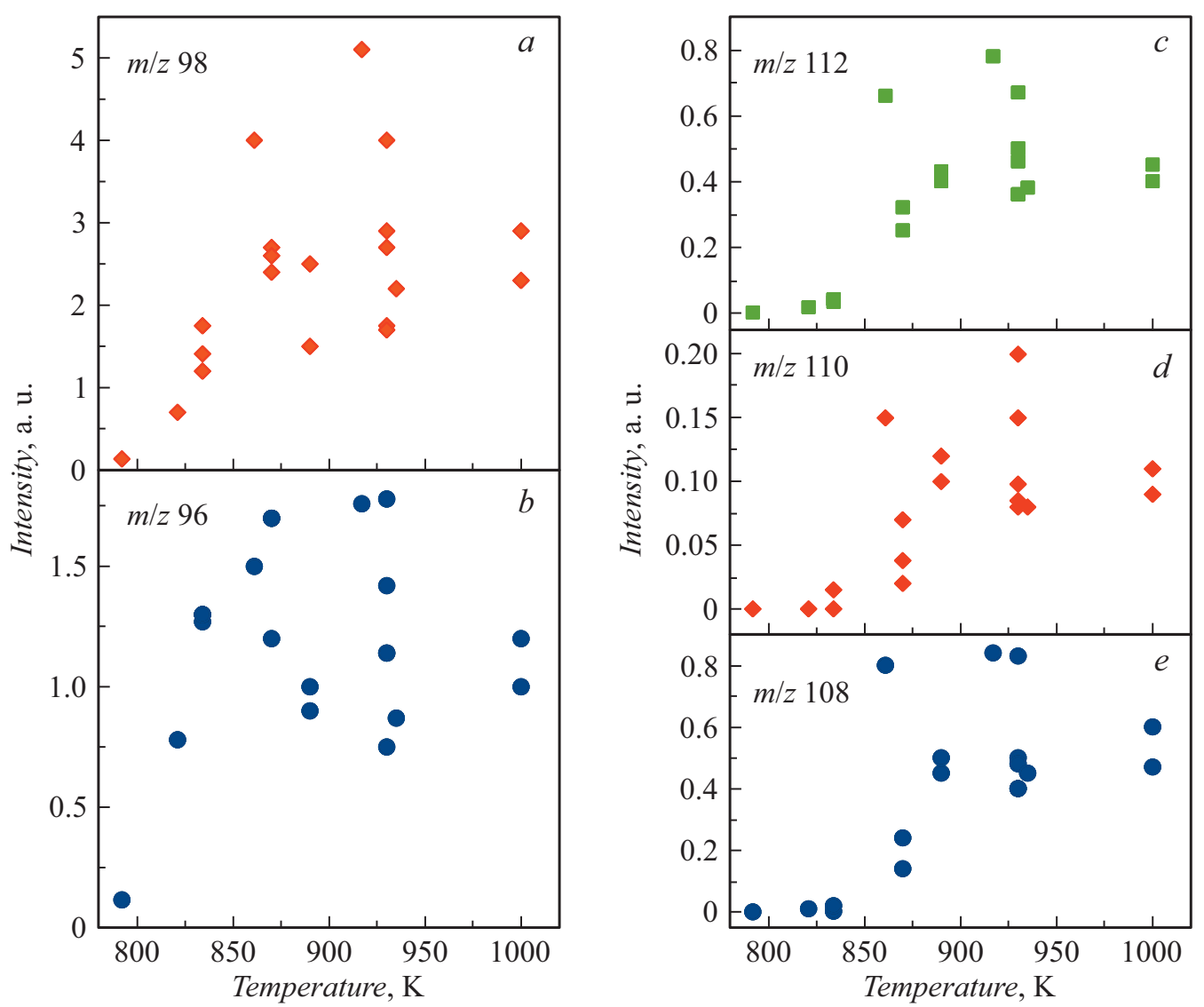

Рис. 5. Температурные зависимости ионов $m / z 96(b), 98(a), 108(c), 110(d)$ и $112(e)$.

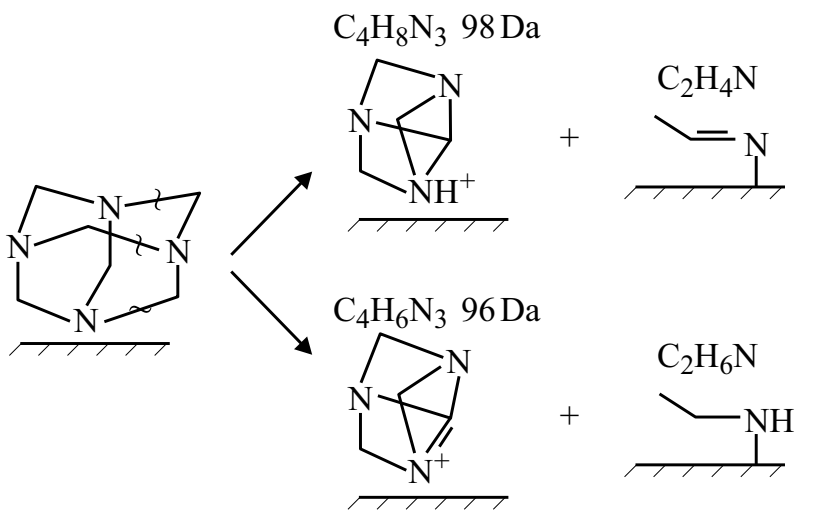

Рис. 6. Схема образования соединений на поверхности $\mathrm{NaAu}_{x}$, приводящих к образованию ионов $m / z, 96$ и 98.

образования состоит в установлении водородной связи между коадсорбированными молекулами, которая может переходить в химическую связь [6,28,29]. Распад димеров адсорбированных молекул приводит к их гидрированию $[6,28]$ и метилированию [18,29]. На рис. 7 показано образование димеров уротропина и некоторые возможные их распады с образованием ионов с $m / z 108,126$, $138,140,154$ и 168 . Ионы с $m / z 152$ образуются так же, как ионы с $m / z 154$, но с $\mathrm{C}=\mathrm{C}$-связью присоединяемой метиленовой группы. Образование димеров свидетельствует о том, что время жизни адсорбированных на поверхности молекул уротропина достаточно велико для эффективного образования димеров.

Температурные зависимости тока ионов с $m / z 106$, $108,126,138,140,152,154$ и 168 близки между собой. У этих зависимостей близкие величины температуры начала регистрации тока и $T_{\max }$. Это свидетельствует о едином механизме их происхождения. Другая группа ионов с $m / z$ 114-107, 85, 58 и 42 имеет более высокие значения температуры начала регистрации тока и $T_{\max }$. Разделение ионов по температурным зависимостям токов ионов в две группы позволяет предположить, что первая группа (низкотемпературная) связана с распадом димеров, а вторая (более высокотемпературная) - с распадом отдельных молекул уротропина. В каждой группе ионизирующиеся соединения различаются по

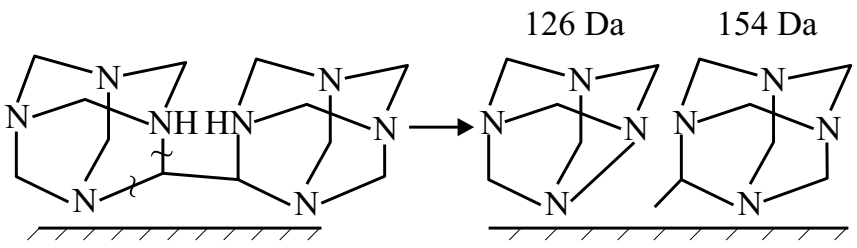

Рис. 7. Схема образования димеров уротропина. 
массе до нескольких раз и соответственно по составу, а также по структуре от „трехмерной“ до „плоской““. Эти различия соединений должны приводить к различию их физико-химических характеристик, в том числе к различию величин $K_{i}$ и $V_{i}$. В соответствии с уравнением (8) это должно проявляться в различии температурных зависимостей, как это наблюдалось для ТИ на металлах и их окислах [1]. Температурные зависимости тока ионов в группе близки, несмотря на отличия их физико-химических характеристик. Это свидетельствует о том, что уравнения (2) и (8) не отражают наблюдаемый процесс ТИ уротропина на $\mathrm{NaAu}_{x}$. Можно предположить, что температурные зависимости тока ионов отражают зависимость эффективности распада адсорбционных комплексов от температуры эмиттера.

Активированные комплексы при накоплении достаточной энергии на связях распадаются с десорбцией продуктов, в том числе в виде ионов. В процессе распада адсорбционного комплекса формируются все характерные для этого распада продукты, регистрируемые в масс-спектре и имеющие подобные температурные зависимости. Это говорит о том, что продукты этих распадов не адсорбируются на поверхности. Первоначальный рост тока ионов с $T$ в двух группах ионов отражает увеличение энергии адсорбционных комплексов с ростом температуры эмиттера. Падение тока ионов при $T>T_{\max }$ свидетельствует о начале распадов комплексов с образованием продуктов, не ионизирующихся в процессе десорбции. Известно, что в диапазоне температур 970-1300 K происходит разложение уротропина с выделением неионизируемых соединений $\mathrm{H}_{2}, \mathrm{CH}_{4}$, $\mathrm{C}_{2} \mathrm{H}_{2}, \mathrm{C}_{2} \mathrm{H}_{4}$ и $\mathrm{C}_{2} \mathrm{H}_{6}$ [30]. Совпадение последовательности распадов молекул уротропина при ЭИ и ТИ и близость температурных зависимостей в группе ионов с $m / z 114-107,85,58$ и 42 может свидетельствовать о том, что в одном акте распада активированного адсорбционного комплекса уротропина происходит вся последовательность распадов, а время жизни энергии активации комплекса достаточно для протекания этих процессов. Из совпадения продуктов при ЭИ и распаде адсорбционного комплекса следует, что эти продукты устойчивы и их образование энергетически выгодно.

Димеры и соответствующие им адсорбционные комплексы могут запасать на $3 n-6$ степенях свободы, где $n$ - число атомов в комплексе, энергии больше, чем молекулы уротропина и соответствующие им комплексы [9]. Благодаря этому группа ионов с $m / z$ 106, $108,126,138,140,154$ и 168, связанная с распадом димеров, имеет более низкие значения температуры начала регистрации тока и $T_{\max }$, чем молекулы.

Для эмиттеров из металлов и окислов характерно установление теплового и зарядового равновесия между твердым телом и адсорбированными на нем органическими соединениями [1], распределение по кинетической энергии десорбирующихся с поверхности ионов отвечает распределению Максвелла при температуре эмиттера $[1,22]$. Если при распаде адсорбцион-

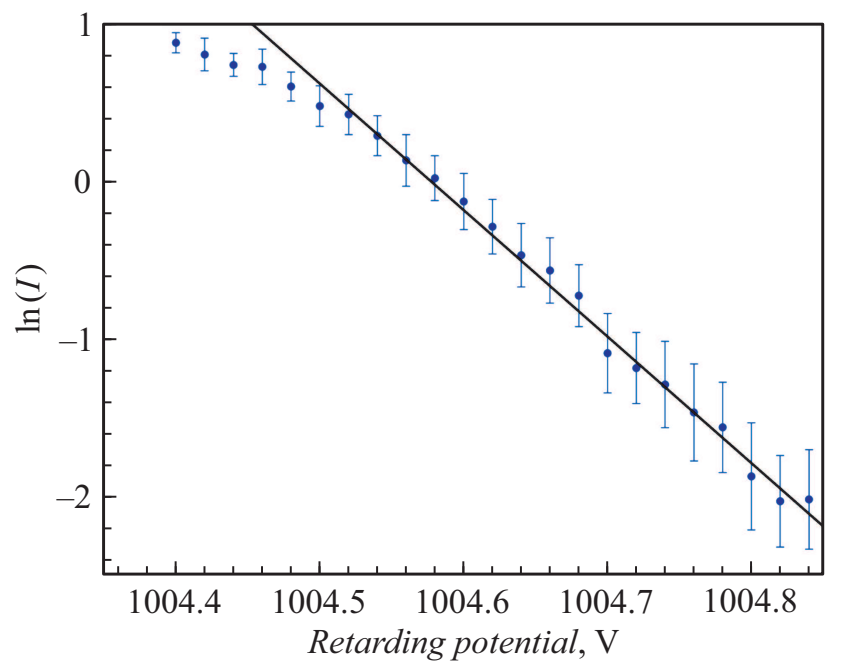

Рис. 8. Логарифмическая зависимость $\ln (I)$ тока ионов с $m / z 98$ от величины задерживающего потенциала. $T$ эмиттера $930 \mathrm{~K}$. Прямая линия - линейная аппроксимация зависимости $\ln (I)$ от $T$.

ных комплексов продукты распада не адсорбируются на поверхности, а десорбируются вследствие перехода на отталкивательную часть потенциальной кривой, то тепловое равновесие между десорбирующимися продуктами распада и твердым телом не устанавливается. Для определения наличия или отсутствия теплового равновесия между продуктами распада уротропина и интерметаллидом $\mathrm{NaAu}_{x}$ выполнено исследование распределения по кинетической энергии ионов, десорбирующихся с поверхности. Его проводили методом кривых задержки путем подачи на электроды источника ЭИ задерживающего потенциала, как это представлено в [19]. На рис. 8 показана характерная логарифмическая зависимость $\ln (I)$ тока ионов с $m / z 98$ от задерживающего потенциала. Ускоряющий потенциал в источнике ТИ составлял $V=1000 \mathrm{~V}$, задерживающий потенциал изменялся в диапазоне $1004-1005 \mathrm{~V}$, что учитывало контактную разницу потенциалов эмиттера и электродов источника ЭИ, на которые подавался задерживающий потенциал. Температура эмиттера составляла $930 \mathrm{~K}$. Погрешность определения температуры эмиттера не превышала 10 градусов.

Наклон прямой на рис. 8, согласно [19,31], определяется, как $a=-1 / k T_{\mathrm{MD}}$ и отвечает температуре распределения Максвелла по кинетической энергии десорбируюшихся с поверхности ионов. Величина $T_{\mathrm{MD}}$ ионов с $m / z, 98$ при ускоряющем поле $1000 \mathrm{~V}$ оставила $T_{\mathrm{MD}}=1350 \mathrm{~K}$, что значительно отличается от температуры эммитера $T=930 \mathrm{~K}$. Погрешность определения наклона прямолинейного участка кривой задержки „, $а$ составляет менее процента, т.е. определение температуры из $a=1 / k T$ составит $(1350 \pm 15) \mathrm{K}$. Температура распределения Максвелла по кинетической энергии $T_{\mathrm{MD}}$ на $420 \mathrm{~K}$ выше, чем температура эмиттера. Это 
свидетельствует о том, что ионы десорбируются с поверхности без достижения теплового равновесия с эмиттером. ТИ уротропина на поверхности $\mathrm{NaAu}_{x}-$ неравновесный процесс и, следовательно, выражение (2) неприменимо. Однако используя температуру $T_{\mathrm{MD}}$ вместо температуры эмиттера для определения $\varphi$ методом полного тока, для молекулярных ионов по (2) получаем $\varphi=6.2-6.5 \mathrm{eV}$, на $1.4 \mathrm{eV}$ ниже, чем оценка в работе [18]. Это значение работы выхода поверхности соответствует значениям $\varphi$ для известных эмиттеров $[1,25]$.

Несоответствие $T_{\mathrm{MD}}$ температуре эмиттера свидетельствует о том, что при распаде активированного адсорбционного комплекса на поверхности $\mathrm{NaAu}_{x}$ десорбция и ионизация частиц происходит в процессе распада комплекса без адсорбции продукта распада на поверхности. Накопленной на степенях свободы комплекса энергии достаточно для разрыва связей, десорбции и ионизации продуктов. Этот механизм обеспечивает высокую эффективность ионизации и приводит к завышению значения работы выхода при ее определении методом полного тока.

\section{Заключение}

Результаты исследования показали, что теория ТИ органических соединений, представленная в обзоре [1], не дает полного описания ионообразования при адсорбции уротропина на поверхности нагретого твердого тела - интерметаллида $\mathrm{NaAu}_{x}$. Время жизни молекул уротропина на поверхности достаточно для эффективного образования димеров уротропина. По температурным зависимостям ионного тока установлено, что наблюдаемые ионы можно разделить на группу ионов, образующихся при ТИ молекул уротропина, и на группу ионов, образующихся при ТИ димеров уротропина. Температура распределения Максвелла по кинетической энергии десорбирующиеся с поверхности ионов превышает температуру эмиттера, что свидетельствует о неравновесности процесса. Рассмотрено предположение, что адсорбированные молекулы и их димеры образуют с поверхностью адсорбционные комплексы, которые за счет накопленной в них энергии распадаются с десорбцией ионов. В процессе распада комплексов реализуется последовательность распадов молекул уротропина, наблюдаемая при электронной ионизации молекул в вакууме. При этом образующиеся в процессе распада адсорбционных комплексов продукты десорбируются без адсорбции на поверхности. Температурные зависимости ионных токов уротропина и продуктов реакций на поверхности с его участием определяются температурной зависимостью активирования соответствующих адсорбционных комплексов.

\section{Финансирование работы}

Исследование выполнено при финансовой поддержке Российского фонда фундаментальных исследований в рамках научного проекта № 20-02-00370.

\section{Конфликт интересов}

Авторы заявляют, что у них нет конфликта интересов.

\section{Список литературы}

[1] U.Kh. Rasulev, E.Ya. Zandberg. Prog. Surf. Sci., 28 (3-4), 181(1988). DOI: 10.1016/0079-6816(88)90003-2

[2] У.Х. Расулев, Э.Я. Зандберг. Жур. аналит. хим., 27 (12), 2459 (1972).

[3] У.Х. Расулев, Э.Я. Зандберг, Ш.М. Халиков. ЖТФ, 66 (4), 832 (1976).

[4] М.В. Кнатько, М.Н. Лапушкин. ЖТФ, 83 (6), 51 (2013). [M.V. Knat'ko, M.N. Lapushkin. Tech. Phys., 58(6), 827 (2013). DOI: 10.1134/S1063784213060170]

[5] М.В. Кнатько, М.Н. Лапушкин, В.И. Палеев. ЖТФ, 74 (7), 99 (2004). [M.V. Knat'ko, M.N. Lapushkin, V.I. Paleev. Tech. Phys., 49 (7), 905 (2004). DOI: 10.1134/1.1778866]

[6] Э.Я. Зандберг, У.Х. Расулев, М.Р. Шарапудинов. ТЭХ, 7 (3), 363 (1971). [E.Ya. Zandberg, U.Kh. Rasulev, M.P. Sharapudinov. Theor. Exp. Chem., 7 (3), 299 (1973). DOI: 10.1007/BF00525532]

[7] У.Х. Расулев, Ш.М. Халиков. ЖОХ, 12 (3), 479 (1976).

[8] Н.М. Блашенков, Н.И. Ионов, Г.Я. Лаврентьев. Письма в ЖТФ, 13 (7), 392 (1987). [N.M. Blashenkov, N.I.Ionov, G.Ya. Lavrent'ev. Sov. Tech. Phys. Lett., 13 (7), 160 (1987).]

[9] В.Н. Кондратьев. Кинетика химических газовых реакций (АН ССCР, М., 1958)

[10] Г.Я. Лаврентьев. ЖТФ, 71 (10), $120 \quad$ (2001). G.Ya. Lavrent'ev. Tech. Phys., 46 (10), 1322 (2001). DOI: $10.1134 / 1.1412071]$

[11] H.L. Abbott, I. Harrison. J. Chem. Phys., 125 (2), 024704 (2006). DOI: $10.1063 / 1.2208362$

[12] K.M. DeWitt, L. Valadez, H.L. Abbott, K.W. Kolasinski, I. Harrison. J. Phys. Chem. B, 110 (13), 6705 (2006). DOI: $10.1021 / \mathrm{jp} 0566865$

[13] J.J. Chen, N. Winograd. Surf. Sci., 326 (3), 285 (1995). DOI: $10.1016 / 0039-6028(94) 00817-5$

[14] J. Liu, C. Lv, C. Jin, Y. Guo, G. Wang. RSC Adv., 5 (26), 20208 (2015). DOI: 10.1039/C4RA16308G

[15] T. Fuji, H. Jimba. Int. J. Mass Spectr. Ion Proces., 79 (3), 221 (1987). DOI: 10.1016/0168-1176(87)83001-X

[16] M.V. Knatko, M.N. Lapushkin. Eur. J. Mass. Spectrom., 26 (5), 351 (2020). DOI: 10.1177/1469066720950553

[17] J.B. Wu, Y.W. Yang, Y.F. Lin, H.T. Chiu. J. Phys. Chem. B, 108 (5), 1677 (2004). DOI: 10.1021/jp0349233

[18] М.В. Кнатько, М.Н. Лапушкин. Масс-спектр., 16 (47), 323 (2019). DOI: 10.25703/MS.2019.16.46 [M.V. Knatko, M.N. Lapushkin. J. Analit. Chem., 16 (4), 323 (2019). DOI: $10.1134 / \mathrm{S} 1061934820140105]$

[19] В.И. Палеев, В.И. Каратаев, Э.Я. Зандберг. ЖТФ, 36 (8), 1459 (1966).

[20] X. Xu, X. Zhang, S. Abbas, E. Karangwa, E. Duhoranimana, F. Peng, P. Shu. Food Control, 57 (1), 89 (2015). DOI: $10.1016 /$ j.foodcont.2015.03.048 
[21] Э.Я. Зандберг, У.Х. Расулев, Ш.М. Халиков. ЖОХ, 10 (6), 1137 (1974).

[22] Э.Я. Зандберг, У.Х. Расулев, М.Р. Шарапудинов. Изв. АН СССР, сер. физ., $35(2), 334$ (1971).

[23] Э.Я.Зандберг, Н.И. Ионов. Поверхностная ионизация (Наука, M., 1969) [E.Ya. Zandberg, N.I. Ionov. Surface Ionization (Israel Program of Scientific Translations, Jerusalem, 1971)]

[24] A.H. Cowley, M. Lattman, P.M. Stricklen. Inorg. Chem., 21 (2), 543 (1982). DOI: 10.1021/ic00132a016

[25] Э.Я. Зандберг, У.Х. Расулев, Э.Г. Назаров. ЖТФ, 51 (6), $1242(1981)$.

[26] А. Холов, К.С. Турсунов, Э.Г. Назаров, М.Н. Лапушкин, М.В. Кнатько, Э.Я. Зандберг, У.Х. Расулев, И.Л. Журавлева. ТЭХ, 20 (4), 434 (1984). [A. Kholov, K.S. Tursunov, U.Kh. Rasulev, É.G. Nazarov, M.N. Lapushkin, M.V. Knat'ko, É.Ya. Zandberg, I.L. Zhuravleva. Theor. Exp. Chem., 20 (4), 405 (1984). DOI: 10.1007/BF00516575]

[27] D.J. Segobia, A.F. Trasarti, C.R. Apesteguía. Chin. J. Catal., 40 (11), 1693 (2018). DOI: 10.1016/S1872-2067(18)63179-1

[28] Э.Я. Зандберг, У.Х. Расулев. ДАН СССР, 187 (4), 777 (1969).

[29] Э.Я. Зандберг, В.И. Палеев, А.Л. Нездюров, Д.А. Пономарев. Теор. и эксп. химия, $24(6), 733$ (1988). [É.Ya. Zandberg, A.L. Nezdyurov, V.I. Paleev, D.A. Ponomarev. Theor. Exp. Chem., 24 (6), 703 (1988).]

[30] Новый справочник химика и технолога. Сырье и продукты промышленности органических и неорганических веществ (НПО Профессионал, СПб, 2002), ч. 1.

[31] Э.Я. Зандберг, Н.И. Ионов, В.И. Палеев, А.Я. Тонтегоде. ЖТФ, 32 (4), 503 (1962). 